\title{
CDISC SEND Rad/Nuc Agent lonizing Radiation Type Response Terminology
}

National Cancer Institute

\section{Source}

National Cancer Institute. CDISC SEND Rad/NuC Agent Ionizing Radiation Type Response

Terminology. NCl Thesaurus. Code C158121.

Terminology associated with the rad/nuc agent ionizing radiation type Response codelist

of the Clinical Data Interchange Standards Consortium (CDISC) Standard for the Exchange of Non-clinical Data (SEND). 